\title{
Effect of oxidation-reduction potential on performance of European sea bass (Dicentrarchus labrax) in recirculating aquaculture systems
}

\author{
Xian $\mathrm{Li}^{1}$, Jean-Paul Blancheton ${ }^{2,3, *}$, Ying Liu ${ }^{1,{ }^{*}}$, Sebastien Triplet $^{2}$, Luigi Michaud $^{4}$
}

\author{
${ }^{1}$ Institute of Oceanology, Chinese Academy of Sciences, Qingdao, 266071, China \\ 2 Ifremer, 34250, Palavas-les-Flots, France \\ ${ }^{3}$ UMR Ecosym, USTL, 34000, Montpellier, France \\ ${ }^{4}$ University of Messina, 98166 , Messina, Italy
}

\begin{abstract}
*: Corresponding authors : Jean-Paul Blancheton, email address : Jean.Paul.Blancheton@ifremer.fr ; Ying Liu, email address : yinliu@ms.qdio.ac.cn
\end{abstract}

\begin{abstract}
:
The direct impact of oxidation-reduction potential (ORP) on fish welfare and water quality in marine recirculating aquaculture systems (RAS) is poorly documented. In this study, the effects of the fish size $\left(\mathrm{S}_{1}, \mathrm{~S}_{2}, \mathrm{~S}_{3}\right)$ and ORP level (normal, four successive levels) on the performance of European sea bass (Dicentrarchus labrax) were investigated. Three size fish were distributed into two RAS (RAS and RAS $\mathrm{O}_{3}$ ). Ozone was injected into RAS $\mathrm{O}_{3}$ to increase the ORP level. The ORP was stabilized to four successive levels: $260-300,300-320,320-350$, and $300-320 \mathrm{mV}$ in fish tanks during four periods $\left(P_{1-4}\right)$. At the last day of each period, the hematological parameters, plasma protein and mortality of sea bass were analyzed. Two-way ANOVA revealed that several hematological parameters, including $\mathrm{pH}$, hematocrit, concentrations of oxygen, carbon dioxide, glucose (Glu), ionized calcium, kalium, and hemoglobin, were significantly influenced by the increased ORP levels over the experimental period. The alteration in blood Glu and plasma protein concentration showed that ORP around $300-320 \mathrm{mV}$ started to stress sea bass. Once the ORP exceeded $320 \mathrm{mV}$ in the tanks during the $\mathrm{P}_{3}$ period, mortality occurred even when total residual oxidants/ozone-produced oxidants was only $0.03-$ $0.05 \mathrm{mg} \mathrm{L}^{-1}$ in the fish tanks. At the same time, plasma protein decreased notably due to appetite depression. After the decrease in ORP during the $\mathrm{P}_{4}$ period, mortality continued. In conclusion, the results strongly suggest that for European sea bass in RAS, the ORP should not exceed $320 \mathrm{mV}$ in the tanks. Once ozonation damaged fish, the effect seemed to be irreversible. However, how ORP affected related hematological parameters still need the further investigations.
\end{abstract}

Keywords: ORP ; Performance ; European sea bass ; RAS ; Ozone

This work was supported by the National Natural Science Foundation of China (Grant No. 41306152) and National Science and Technology Support Program (Grant No. 2011BAD13B04). This article is also a part of cooperation between China (IOCAS), France (Ifremer), and Italy (University of Messina). 


\section{Introduction}

With limited land and clean seawater resources, and increasing demand for seafood, the expanding aquaculture industry is facing many challenges (Zhang et al. 2011). In response, recirculating aquaculture systems (RAS) or semi-RAS are frequently used instead of flow-though systems (FTS). Compared with traditional FTS, RAS offer advantages such as a stable environment in terms of physico-chemical parameters and microbial flora, which provides an effective control or pre-control of diseases and improves the welfare of culture animals and less environmental impact (Blancheton 2000; Tal et al. 2009).

Many studies have focused on optimizing the RAS environment, especially physicochemical indexes, in order to optimize the living conditions of cultured animals. The impacts of water quality parameters such as $\mathrm{pH}$, temperature, salinity, and dissolved gas in RAS on the biological performance of fish have been studied (Kristensen et al. 2009; Soria et al. 2007). Seawater is a complex medium with strong interactions among chemical parameters, as $\mathrm{pH}$ interacts with the gas balance (mainly $\mathrm{CO}_{2}$ ), and $\mathrm{pH}$ and temperature affect the balance between un-ionized and ionized ammonia (Sanni and Forsberg 1996; Whitfield 1974).

The oxidation-reduction potential (ORP) results from all reactions involving both oxidations and reductions and varies as a function of the standard potential, relative ion concentration, temperature, and the number of electrons transferred (Banhidi 1995). It is dependent on all oxidants and reducers present in the system. In water, the ORP is strongly related to temperature, $\mathrm{pH}$, salinity, and concentrations of dissolved oxygen and dissolved oxidants such as ozone (Liu et al. 2009; Summerfelt et al. 2009; Tango and Gagnon 2003). Nowadays, ozone is wildly used in aquaculture for disinfection, water treatment, and bacteria control (Buchan et al. 2006; Forneris et al. 2003).

During ozonation, ozone reacts with several compounds resulting in the formation of -ozone-produced oxidants" (OPO) also mentioned as total residual oxidants (TRO) including free bromine $(\mathrm{HOBr} / \mathrm{OBr}-)$ and bromamines $(\mathrm{NH} 2 \mathrm{Br}, \mathrm{NHBr} 2)$, which are more stable than ozone. The toxicity of TRO and particularly of related bromate compounds to aquatic animals at low dosage is focused (Reiser et al. 2010). However it is also proved that bromate will not be formed when ammonia is present in the seawater (Tango et al. 2003, Schroeder et a. 2011).

To date, few studies mentioned a direct connection between ORP and biology, although most existing reports describe the ORP as an easy tool for monitoring ozonation in aquaculture systems (Buchan et al. 2005). Summerfelt et al (2009) reported that an ORP of $375-525 \mathrm{mV}$ was required to reach the mean daily ozone concentration necessary to obtain full-flow disinfection in freshwater RAS. For southern rock lobster larvae, survival was higher and bacterial contamination was 
lower when the ORP was between 330 and $500 \mathrm{mV}$ (Ritar et al. 2006). With moderate ozonation corresponding to an ORP value of $250 \mathrm{mV}$ in a low exchange freshwater RAS, rainbow trout showed improved performance compared to a system without ozonation (Good et al. 2011). However, the influence of ORP modification using ozonation on marine fish physiology in RAS has rarely been studied (Silva et al. 2011). In particular little is known about European sea bass which is widely cultured in the Mediterranean Sea (Blancheton 2000).

In this study, the impacts of (1) four successive ORP levels induced by ozonation and (2) fish sizes on physiological and hematological parameters of European sea bass (Dicentrarchus labrax) were studied and compared to data from a RAS without ozonation. The goals of this study were to identify the safe range of ORP for sea bass and to evaluate how European sea bass respond to ORP alterations.

\section{Materials and method}

\subsection{RAS structure}

Two RAS located at the French Research Institute for Exploration of the Sea (Ifremer, Palavas les Flots, Montpellier, France) have been running for several years. Each consists of three $1 \mathrm{~m}^{3}$ fish tanks, a mechanical filter, UV disinfection, a protein skimmer, a warm-cold exchanger, moving bed biofilters, a degassing unit, and storage and pumping tanks organized in four loops (Fig. 1). In the first loop, water flowing out of the fish tank is filtered in a mechanical filter and pumped from the storage tank back to the fish tanks. Three parallel loops connected to the pumping tank were used for the other water treatments (1) biofiltration, (2) ozonation and protein skimming and (3) UV disinfection, a heat exchange, and degassing. The first of the two RAS was equipped with an $\mathrm{O}_{3}$ generator (BMT 802N, BMT Messtechnik, Berlin, Germany), which converted pure oxygen to ozone. This system was named $\mathrm{RAS} \mathrm{O}_{3}$. The $\mathrm{O}_{3}$ generator was controlled by an ORP sensor placed after the biofilters and delivered a maximum of $4 \mathrm{~g} \mathrm{O}_{3}$ per hour. In the second system, named RAS, air was used as feed gas in the skimmer. Two systems were set up in the same way before ozone injection into RAS $\mathrm{O}_{3}$.

$\mathrm{pH}$ was controlled by injecting sodium hydroxide into the pumping tank in order to keep the value around $7.2-7.8$ in the fish tanks. Temperature was maintained at $21.5^{\circ} \mathrm{C}$. Salinity was between 20-30 ppt. Oxygen concentrations in all fish tanks of both systems were almost similar at a super saturation level between $120-140 \%$ except it in fish tanks of RAS $\mathrm{O}_{3}$ was $10 \%$ higher than RAS in the first two days at the $\mathrm{P}_{3}$ period. The water flow rate to each fish tank was $1 \mathrm{~m}^{3} \mathrm{~h}^{-1}$. The daily water renewal rate was between 1 and $2 \mathrm{~m}^{3}$ of new water per $\mathrm{kg}$ feed. 


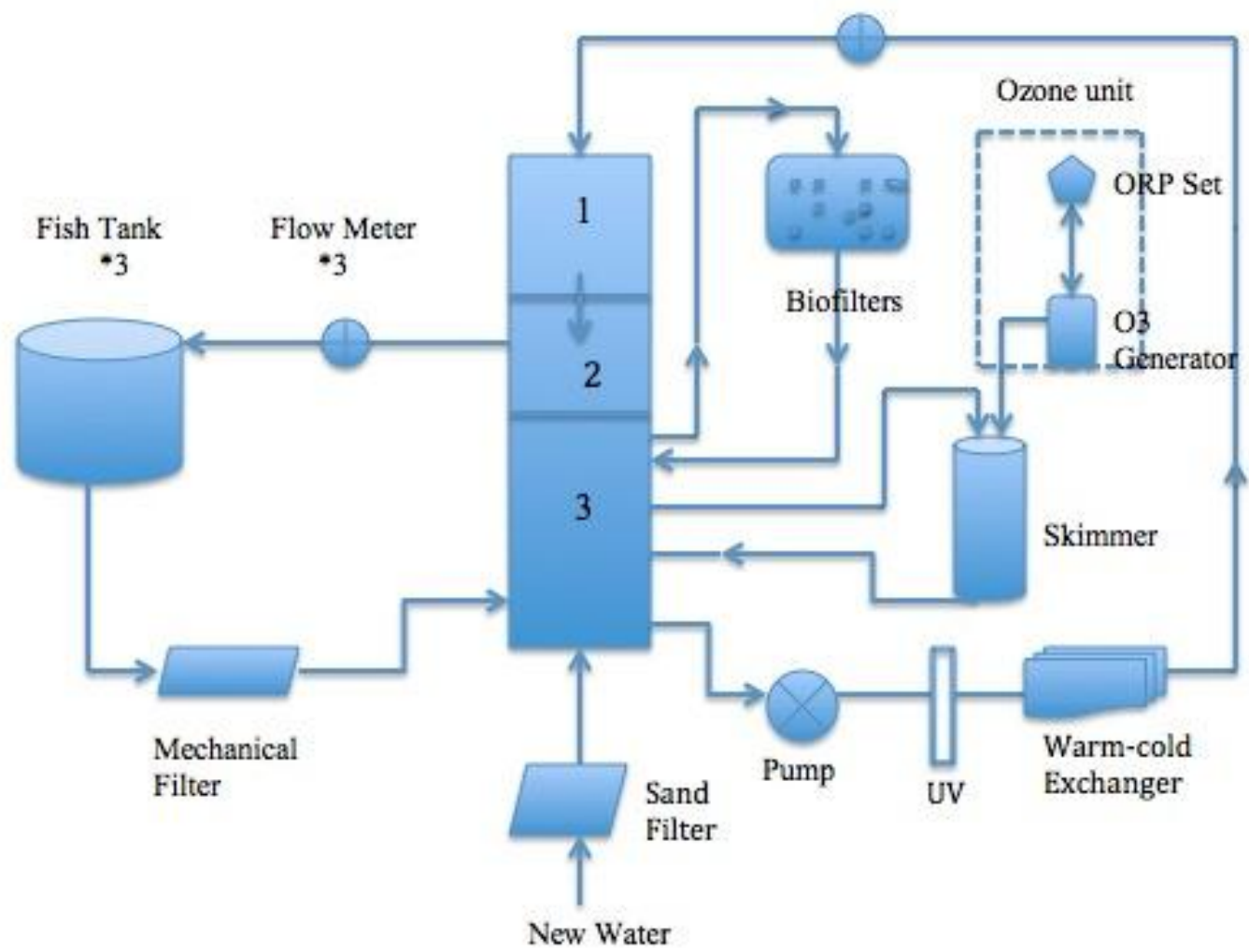

1. Degassing Unit; 2. Storage Tank; 3. Pumping Tank

Fig 1. The structure of RAS O3

\subsection{ORP levels control}

The ORP was stabilized at four successive levels: $260-300 \mathrm{mV}, 300-320 \mathrm{mV}, 320$ $350 \mathrm{mV}$ and $300-320 \mathrm{mV}$ in fish tanks during four periods $\left(P_{1-4}\right)$ as shown on Table 1. During the $\mathrm{P}_{1}$ period, the ORP level was increased gradually over 4 days to reach $\sim 290$ $\mathrm{mV}$ in the fish tanks, and this value was maintained for 8 days. During the $\mathrm{P}_{2}$ period, the ORP in the fish tanks was between 300 and $320 \mathrm{mV}$. During the $P_{3}$ period, the ORP in the fish tanks was $320-350 \mathrm{mV}$. Three days after the ORP reached $330 \mathrm{mV}$, mortality occurred. Two days later, ORP was decreased to the same level as that of the $P_{2}$ period $(320 \mathrm{mV})$, and it was maintained for 15 days during the $P_{4}$ period. 
Table 1 : ORP levels and oxygen concentrations in fish tanks during four periods in both RAS $\mathrm{O}_{3}$ and RAS

\begin{tabular}{lccccc}
\hline Period & \multicolumn{3}{c}{$\mathrm{RAS} \mathrm{O}_{3}$} & RAS \\
\cline { 2 - 6 } & $\mathrm{P}_{1}$ & $\mathrm{P}_{2}$ & $\mathrm{P}_{3}$ & $\mathrm{P}_{4}$ & $\mathrm{P}_{1}-\mathrm{P}_{4}$ \\
\hline Duration & 12 & 29 & 13 & 15 & 69 \\
ORP $(\mathrm{mv})$ & $260-300$ & $300-320$ & $320-350$ & $300-320$ & $250-270$ \\
TRO $\left(\mathrm{mg} \mathrm{L}^{-1} \mathrm{cl}\right)$ & ------- & ------- & $0.03-0.05$ & ------- & ------- \\
\hline
\end{tabular}

----------: below detectable limits $\left(0.02 \mathrm{mg} \mathrm{L}^{-1}\right)$

\subsection{Acclimation and feeding of fish}

European sea bass were reared at Ifremer. They were first stored in one big tank for several months. Two months before the trial, they were transferred to the RAS. During this time they were fed at $1.0-1.5 \%$ of the biomass per day.

\subsection{Stocking density and distribution of fish}

Before distribution into the tanks, fish were starved for $24 \mathrm{~h}$ and anaesthetized using eugenol (40 $\left.\mathrm{mg} \mathrm{L}^{-1}\right)$. Three fish sizes were selected as follows: S1: $100 \mathrm{~g}$; S2: $150 \mathrm{~g}$; and S3: $200 \mathrm{~g}$. Each size class was distributed into one of the three tanks in each of the two RAS at a stocking density of $20 \mathrm{~kg} \mathrm{~m}^{-3}$ (Table 2). During the experiment, fish were fed Ep 3-5 dry pellets (Le Gouessant, Brittany, France) at 1.0-1.5\% of biomass per day using auto feeders. The ingested feed quantity was calculated from the feed trap.

Table 2 : Distribution of three sizes classes in RAS and RAS $\mathrm{O}_{3}$

\begin{tabular}{|c|c|c|c|c|c|c|}
\hline & \multicolumn{3}{|c|}{ RAS } & \multicolumn{3}{|c|}{ RAS O3 } \\
\hline & S1 & $\mathrm{S} 2$ & S3 & S1 & S2 & S3 \\
\hline $\begin{array}{l}\text { Mean } \\
\text { weight } \\
\text { (g) }\end{array}$ & $107.24 \pm 14.28$ & $150.36 \pm 13.74$ & $201.6 \pm 26.70$ & $106.74 \pm 14.04$ & $152.67 \pm 14.28$ & $201.6 \pm 26.62$ \\
\hline $\begin{array}{l}\text { Density } \\
\left(\mathrm{kg} \mathrm{m}^{-3}\right)\end{array}$ & 20.05 & 20.15 & 20.2 & 20.07 & 20.00 & 20.2 \\
\hline
\end{tabular}

\subsection{Measured parameters of water quality}

The temperature, salinity, $\mathrm{pH}$, and oxygen concentration in the fish tanks were monitored and recorded daily using an ODEON device (Ponsel, France). Water samples from the fish tanks were collected at 10:00 am twice a week and stored at -20 
${ }^{\circ} \mathrm{C}$ after being filtration on GF/C porous membrane. TAN (total ammonia nitrogen), $\mathrm{NO}_{2}-\mathrm{N}$ (nitrite-nitrogen) and $\mathrm{PO}_{4}-\mathrm{P}$ (orthophosphates) were measured using an Alliance Futura instrument (Sociele AMS France, Frepillon, France) mentioned as Sammouth et al (2009). Total suspended solid (TSS) concentration in the fish tanks was determined twice a week after filtration on GF/C porous membrane; data were expressed in the unit of $\mathrm{mg}$ per $1 \mathrm{~L}$ water. Total resident ozone (TRO equal to OPO) in the fish tanks was measured three times a week at the $P_{1}, P_{2}, P_{4}$ periods while three times a day at the $\mathrm{P}_{3}$ period using the colorimetric N,N-diethyl-p-phenylenediamine (DPD) method (Aqualytic, Langen, German). Its concentration was expressed as $\mathrm{mg}$ $\mathrm{L}^{-1}$ of chlorine.

All fish were starved for $24 \mathrm{~h}$ before sampling. At the last day of each period, fish were removed from the tanks and blood was taken from the caudal vein with syringes within $30 \mathrm{~s}$. Immediately, one or two drops of the blood were used for hematological parameter measurements using an I-STAT portable analyzer (Abbott Laboratories, Abbott Park, IL, USA) equipped with CG8+ cartridges (Good et al. 2011). The pH, $\mathrm{pCO}_{2}, \mathrm{pO}_{2}, \mathrm{HCO}_{3}$, total $\mathrm{CO}_{2}\left(\mathrm{TCO}_{2}\right), \mathrm{O}_{2}$ saturation $\left(\mathrm{sO}_{2} \%\right)$, sodium $(\mathrm{Na})$, potassium $(\mathrm{K})$, ionized calcium (iCa), glucose ( $\mathrm{Glu})$, hematocrit ( $\mathrm{Hct}$ ), and hemoglobin $(\mathrm{Hb})$ of the whole blood were measured. The remaining blood was placed in dry heparinized Eppendorf tubes and centrifuged for $15 \mathrm{~min}$ at $5000 \mathrm{rpm}$ at $4{ }^{\circ} \mathrm{C}$. The upper plasma was collected in the Eppendorf tube and stored at $-80{ }^{\circ} \mathrm{C}$ until analysis. At the end of $P_{1}$ and $P_{2}$ period, four fish more from $S_{1}$ group and one fish more from $S_{2}$ group were removed to avoid density interference.

The concentration of protein in the plasma was determined by spectrophotometer using a kit (BCA Protein Assay Kit, Thermo Scientific, Hudson, New Hampshire, USA). Based on the reaction of protein with alkaline $\mathrm{Cu}^{2+}$, the plasma was first diluted 2000 times in a four-step operation to obtain the best test range. Tests were performed, and run in duplicate (Smith et al. 1985).

Since the $\mathrm{P}_{3}$ period, some fish in bad status were lying on the bottom. The fish sampled in RAS $\mathrm{O}_{3}$ at the $\mathrm{P}_{3}$ and $\mathrm{P}_{4}$ periods were only swimming.

\subsection{Data analysis}

All results were expressed as mean \pm S.D. All statistical analyses were performed using SPSS 16.0 for Windows. The data were first tested for homogeneity using Levene's $F$-test. The percent data were analyzed after arcsine transformation. The differences in water quality parameters between RAS $\mathrm{O}_{3}$ and RAS at the $\mathrm{P}_{1}$ and $\mathrm{P}_{2}$ periods were evaluated using paired $t$-tests. The differences in hematological parameters and plasma protein were compared using a two-way ANOVA (fish size, ORP and fish size ${ }^{*} \mathrm{ORP}$ ). Differences were considered statistically significant at $p$ $<0.05$. 


\section{Results}

\subsection{Water quality}

There was no significant difference on all water parameter concentrations between the RAS and RAS $\mathrm{O}_{3}$ groups during the $\mathrm{P}_{1}$ and $\mathrm{P}_{2}$ periods $(p>0.05)$ (Table 3$)$. During these periods, the TSS concentration in the RAS $\mathrm{O}_{3}$ fish tanks was slightly higher than that in the RAS tanks, but the difference was not significant $(p>0.05)$. The TSS and TAN concentrations of the RAS $\mathrm{O}_{3}$ group peaked during the $\mathrm{P}_{2}$ period and then decreased during the $\mathrm{P}_{3}$ and $\mathrm{P}_{4}$ periods.

Table 3. Water parameters $\left(\mathrm{mg} \mathrm{L}^{-1}\right)$ in fish tanks of RAS and RAS $\mathrm{O}_{3}$ during four periods

\begin{tabular}{|c|c|c|c|c|c|c|c|c|}
\hline & \multicolumn{4}{|c|}{ RAS } & \multicolumn{4}{|c|}{ RAS O3 } \\
\hline & $\mathrm{P} 1$ & $\mathrm{P} 2$ & P3 & $\mathrm{P} 4$ & $\mathrm{P} 1$ & $\mathrm{P} 2$ & P3 & $\mathrm{P} 4$ \\
\hline TSS & $6.33 \pm 7.64$ & $6.91 \pm 7.50$ & $6.91 \pm 7.43$ & $6.91 \pm 1.80$ & $9.21 \pm 6.0$ & $17.10 \pm 9.6$ & $9.10 \pm 8.6$ & $6.64 \pm 1.47$ \\
\hline TAN & $0.54 \pm 0.12$ & $0.73 \pm 0.11$ & $0.71 \pm 0.24$ & $0.78 \pm 0.12$ & $0.51 \pm 0.13$ & $0.71 \pm 0.12$ & $0.68 \pm 0.21$ & $0.54 \pm 0.24$ \\
\hline $\mathrm{NO}_{2}-\mathrm{N}$ & $0.04 \pm 0.02$ & $0.05 \pm 0.01$ & $0.06 \pm 0.02$ & $0.07 \pm 0.02$ & $0.04 \pm 0.01$ & $0.05 \pm 0.01$ & $0.05 \pm 0.02$ & $0.04 \pm 0.03$ \\
\hline $\mathbf{P O}_{4}-\mathbf{P}$ & $0.70 \pm 0.12$ & $0.95 \pm 0.11$ & $1.01 \pm 0.13$ & $0.89 \pm 0.24$ & $0.66 \pm 0.34$ & $0.89 \pm 0.21$ & $0.89 \pm 0.22$ & $0.67 \pm 0.33$ \\
\hline
\end{tabular}

Two-paired t-test

\subsection{Mortality}

There were no dead fish in RAS group during $\mathrm{P}_{1-4}$ periods. Although the ORP was set back to 300-320 mv during the $\mathrm{P}_{4}$ period, mortality during $\mathrm{P}_{4}$ was two times higher than that during $P_{3}$ (Fig. 2). The mortality of medium size fish $\left(S_{2}\right)$ was slightly higher than that of the two other sizes, whereas mortality of the largest-sized fish $\left(\mathrm{S}_{3}\right)$ was the lowest, especially during the $\mathrm{P}_{4}$ period.

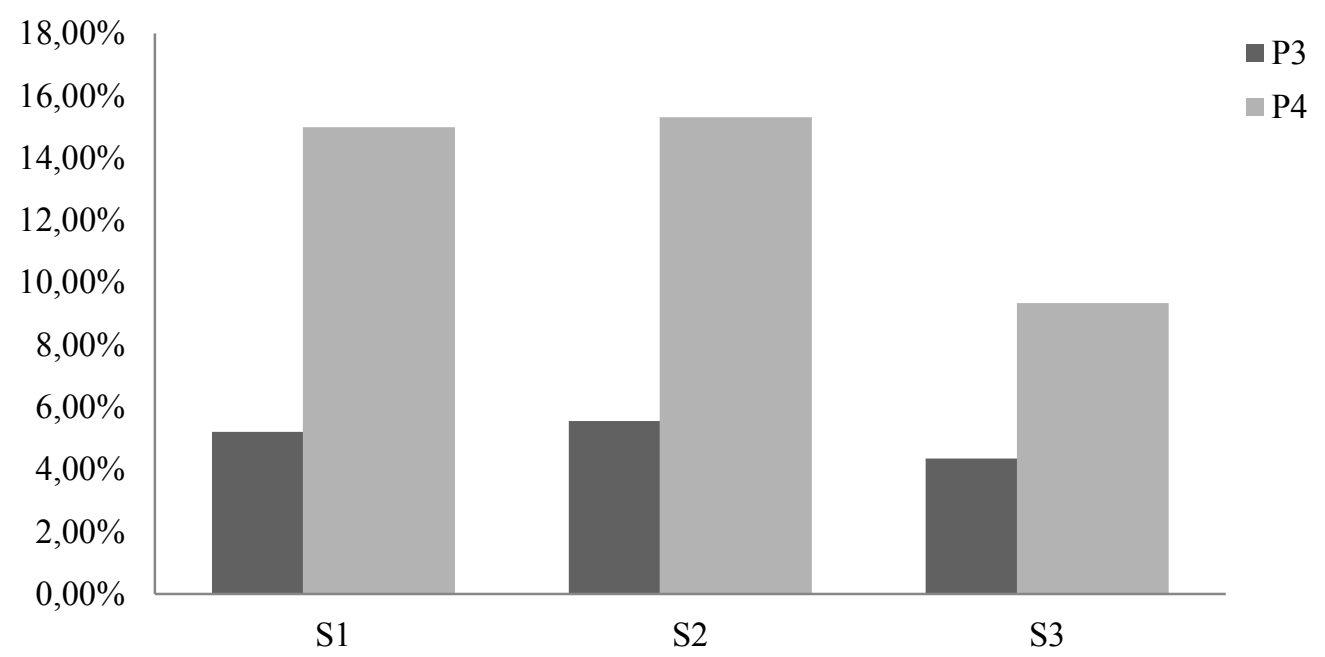

Fig. 2 : Mortalities of three sizes fish in RAS $\mathrm{O}_{3}$ during the $\mathrm{P}_{3}$ and $\mathrm{P}_{4}$ periods 


\subsection{Hematological parameters}

\subsection{1. $\mathrm{P}_{1}$ period}

At the end of the $\mathrm{P}_{1}$ period, blood $\mathrm{O}_{2}$ concentration, measured as $\mathrm{PO}_{2}$ and $\mathrm{sO}_{2} \%$, was first significantly influenced by the increased ORP $(p<0.05)$ (Table 4). At the same time, $\mathrm{Hct}$ and $\mathrm{Hb}$ were lower in both the $\mathrm{S}_{1}$ and $\mathrm{S}_{2}$ groups of RAS $\mathrm{O}_{3}$ and significantly affected by ORP $(p<0.05)$. Besides ORP had a significant effect on iCa concentration.

No effects of fish size and ORP/fish size interaction on related parameters wererecorded $(p>0.05)$ (Table 5).

Table 4 : Hematological parameters of three size classes in RAS and RAS $\mathrm{O}_{3}$ during the $\mathrm{P}_{1}$ period

\begin{tabular}{|c|c|c|c|c|c|c|}
\hline \multirow[t]{2}{*}{ Parameters } & \multicolumn{2}{|l|}{$S 1$} & \multicolumn{2}{|l|}{$S 2$} & \multicolumn{2}{|l|}{ S3 } \\
\hline & $R A S$ & $R A S \quad O 3$ & $R A S$ & $R A S O 3$ & $R A S$ & $R A S O 3$ \\
\hline pH & $7.33 \pm 0.05$ & $7.30 \pm 0.05$ & $7.34 \pm 0.18$ & $7.33 \pm 0.06$ & $7.32 \pm 0.02$ & $7.34 \pm 0.03$ \\
\hline $\mathrm{pCO}_{2}\left(\mathrm{mmol}^{-1)}\right.$ & $19.27 \pm 0.84$ & $22.02 \pm 1.00$ & $18.30 \pm 1.67$ & $20.48 \pm 4.21$ & $20.10 \pm 2.08$ & $17.95 \pm 1.17$ \\
\hline $\mathrm{pO}_{2}(\mathbf{m m H g})$ & $44.75 \pm 2.28$ & $54.75 \pm 2.86$ & $43.50 \pm 2.38$ & $56.00 \pm 5.48$ & $47.75 \pm 12.42$ & $46.00 \pm 4.08$ \\
\hline $\mathrm{HCO}_{3}\left(\mathrm{mmolL}^{-1}\right)$ & $10.43 \pm 1.20$ & $10.10 \pm 0.19$ & $09.98 \pm 0.71$ & $10.40 \pm 0.43$ & $10.43 \pm 1.06$ & $9.75 \pm 0.21$ \\
\hline $\mathrm{tCO}_{2}\left(\mathrm{mmol} \mathrm{L}^{-1}\right)$ & $11.00 \pm 1.22$ & $10.75 \pm 0.43$ & $10.5 \pm 0.58$ & $11.00 \pm 2.00$ & $10.75 \pm 0.56$ & $10.25 \pm 0.50$ \\
\hline $\mathrm{sO}_{2}(\%)$ & $78.25 \pm 4.26$ & $85.25 \pm 0.83$ & $78.25 \pm 2.37$ & $87.00 \pm 2.94$ & $78.75 \pm 11.93$ & $80.75 \pm 3.86$ \\
\hline $\mathrm{Na}\left(\mathrm{mmol} \mathrm{L}^{-1}\right)$ & $160.50 \pm 2.60$ & $157.75 \pm 3.27$ & $159.50 \pm 1.73$ & $155.50 \pm 2.89$ & $159.00 \pm 3.92$ & $160.25 \pm 2.87$ \\
\hline$K\left(\mathrm{mmol} \mathrm{L}^{-1}\right)$ & $3.90 \pm 0.19$ & $4.15 \pm 0.32$ & $3.95 \pm 0.19$ & $3.70 \pm 0.72$ & $4.03 \pm 0.10$ & $3.63 \pm 0.17$ \\
\hline iCa $\left(\mathrm{mmol} \mathrm{L}^{-1}\right)$ & $1.34 \pm 0.07$ & $1.49 \pm 0.03$ & $1.37 \pm 0.08$ & $1.45 \pm 0.03$ & $1.41 \pm 0.09$ & $1.48 \pm 0.08$ \\
\hline Glu $\left(\mathrm{mg} \mathrm{dL}^{-1}\right)$ & $70.50 \pm 5.02$ & $68.75 \pm 1.78$ & $67.50 \pm 7.59$ & $63.50 \pm 7.72$ & $74.50 \pm 13.60$ & $71.75 \pm 10.87$ \\
\hline Het (\% PCV) & $23.75 \pm 2.59$ & $18.85 \pm 2.17$ & $20.75 \pm 3.50$ & $15.50 \pm 1.50$ & $22.00 \pm 5.48$ & $19.00 \pm 5.48$ \\
\hline $\mathrm{Hb}\left(\mathrm{g} \mathrm{dL}^{-1}\right)$ & $8.05 \pm 0.88$ & $6.40 \pm 0.75$ & $7.08 \pm 1.15$ & $5.10 \pm 0.34$ & $7.48 \pm 1.89$ & $6.45 \pm 1.85$ \\
\hline
\end{tabular}


Table 5 : Two-way ANOVA analysis of ORP, fish size and ORP* fish size on related hematological parameters

\begin{tabular}{|c|c|c|c|c|c|c|c|c|c|c|c|c|}
\hline \multirow[t]{2}{*}{ Indice } & \multicolumn{4}{|c|}{ ORP } & \multicolumn{4}{|c|}{ Fish Size } & \multicolumn{4}{|c|}{ ORP*Fish Size } \\
\hline & $\mathrm{P} 1$ & $\mathrm{P} 2$ & P3 & $\mathrm{P} 4$ & $\mathrm{P} 1$ & $\mathrm{P} 2$ & P3 & $\mathrm{P} 4$ & $\mathrm{P} 1$ & $\mathrm{P} 2$ & P3 & $\mathrm{P} 4$ \\
\hline $\mathrm{pH}$ & + & +++ & +++ & +++ & + & ++ & ++ & + & + & +++ & ++ & ++ \\
\hline $\mathrm{pCO}_{2}\left(\mathrm{mmol} \mathrm{L}^{-1}\right)$ & + & +++ & +++ & +++ & + & + & + & + & + & + & +++ & +++ \\
\hline $\mathrm{pO}_{2}(\mathrm{mmHg})$ & ++ & +++ & +++ & +++ & + & ++ & + & +++ & + & +++ & +++ & +++ \\
\hline $\mathrm{HCO}_{3}\left(\mathrm{mmolL}^{-1}\right)$ & + & +++ & +++ & +++ & + & ++ & + & +++ & + & +++ & + & + \\
\hline $\mathrm{tCO}_{2}\left(\mathrm{mmol} \mathrm{L}^{-1}\right)$ & + & ++ & +++ & +++ & + & + & + & ++ & + & +++ & + & + \\
\hline $\mathrm{SO}_{2}(\%)$ & ++ & +++ & +++ & +++ & + & ++ & + & ++ & + & +++ & +++ & + \\
\hline $\mathrm{Na}\left(\mathrm{mmol} \mathrm{L}^{-1}\right)$ & + & + & + & + & + & + & ++ & ++ & + & + & + & + \\
\hline $\mathrm{K}\left(\mathrm{mmol} \mathrm{L}{ }^{-1}\right)$ & + & +++ & +++ & +++ & + & +++ & + & + & + & ++ & + & + \\
\hline $\mathrm{iCa}\left(\mathrm{mmol} \mathrm{L}^{-1}\right)$ & +++ & + & + & + & + & + & + & + & + & + & + & + \\
\hline Glu $\left(\mathrm{mg} \mathrm{dL}^{-1}\right)$ & + & +++ & +++ & +++ & + & +++ & +++ & ++ & + & +++ & +++ & +++ \\
\hline Hct $(\% \mathrm{PCV})$ & ++ & ++ & ++ & +++ & + & + & + & ++ & + & + & ++ & ++ \\
\hline $\mathrm{Hb}\left(\mathrm{g} \mathrm{dL}^{-1}\right)$ & ++ & +++ & ++ & +++ & + & + & +++ & +++ & + & + & +++ & +++ \\
\hline
\end{tabular}

+ : difference is not significant ( $\mathrm{p}>0.05)$; ++ : difference is significant $(0.01 \leq \mathrm{p}<0.05)$; +++ : difference is extremely different $(\mathrm{p}<0.01)$

\subsection{2. $\mathrm{P}_{2}$ period}

At the end of the $\mathrm{P}_{2}$ period, fish in $\mathrm{RAS} \mathrm{O}_{3}$ had a higher blood oxygen, lower blood $\mathrm{CO}_{2}$, lower $\mathrm{Hct}$ and $\mathrm{Hb}$ compared with same size in RAS. Besides, fish in RAS $\mathrm{O}_{3}$ showed a higher blood $\mathrm{Na}$ and Glu (Table 6).

Two-way ANOVA analysis revealed that statistically significant effects of ORP ( $p<$ 0.05 ) on most hematological parameters, including $\mathrm{pH}, \mathrm{pCO}_{2}, \mathrm{pO}_{2}, \mathrm{HCO}_{3}, \mathrm{tCO}_{2}, \mathrm{sO}_{2}$, $\mathrm{K}$, Glu, Hct and $\mathrm{Hb}$ (Table 5). Fish size had significant effects $(\mathrm{p}<0.05)$ on $\mathrm{pH}, \mathrm{pO}_{2}$, $\mathrm{HCO}_{3}, \mathrm{sO}_{2}, \mathrm{~K}$ and Glu. In addition, fish size and ORP interaction had significant impact on $\mathrm{pH}, \mathrm{pO}_{2}, \mathrm{tCO}_{2}, \mathrm{sO}_{2}, \mathrm{~K}$ and $\mathrm{Glu}$ concentrations. 
Table 6 : Hematological parameters of three size classes in RAS and RAS $\mathrm{O}_{3}$ during the $\mathrm{P}_{2}$ period

\begin{tabular}{|c|c|c|c|c|c|c|}
\hline \multirow[t]{2}{*}{ Parameters } & \multicolumn{2}{|l|}{ S1 } & \multicolumn{2}{|l|}{$S 2$} & \multicolumn{2}{|l|}{$S 3$} \\
\hline & $R A S$ & $R A S \quad O 3$ & $R A S$ & RASO3 & $R A S$ & RASO3 \\
\hline pH & $7.33 \pm 0.03$ & $7.35 \pm 0.04$ & $7.32 \pm 0.04$ & $7.42 \pm 0.06$ & $7.38 \pm 0.01$ & $7.34 \pm 0.04$ \\
\hline $\mathrm{pCO}_{2}\left(\mathrm{mmol} \mathrm{L}^{-1)}\right.$ & $22.36 \pm 1.05$ & $20.31 \pm 1.36$ & $24.65 \pm 1.39$ & $19.51 \pm 1.21$ & $23.03 \pm 3.97$ & $18.98 \pm 1.28$ \\
\hline $\mathrm{pO}_{2}(\mathrm{mmHg})$ & $46.38 \pm 3.78$ & $53.25 \pm 1.90$ & $40.17 \pm 2.86$ & $74.67 \pm 2.88$ & $53.00 \pm 2.31$ & $85.75 \pm 2.63$ \\
\hline $\mathrm{HCO}_{3}\left(\mathrm{mmolL}^{-1}\right)$ & $11.91 \pm 0.56$ & $11.26 \pm 0.80$ & $11.88 \pm 0.70$ & $13.10 \pm 1.27$ & $13.30 \pm 0.73$ & $11.50 \pm 0.77$ \\
\hline $\mathrm{tCO}_{2}\left(\mathrm{mmol} \mathrm{L}^{-1}\right)$ & $13.00 \pm 0.53$ & $11.75 \pm 1.03$ & $12.33 \pm 1.21$ & $13.33 \pm 1.37$ & $14.00 \pm 1.15$ & $12.25 \pm 0.96$ \\
\hline $\mathrm{SO}_{2}(\%)$ & $77.63 \pm 5.63$ & $85.63 \pm 2.83$ & $72.33 \pm 6.15$ & $96.00 \pm 0.89$ & $88.25 \pm 1.26$ & $90.00 \pm 4.90$ \\
\hline $\mathrm{Na}\left(\mathrm{mmol} \mathrm{L}^{-1}\right)$ & $161.88 \pm 2.10$ & $164.13 \pm 2.53$ & $162.00 \pm 3.10$ & $163.17 \pm 2.40$ & $159.5 \pm 1.29$ & $162.25 \pm 3.40$ \\
\hline $\mathrm{K}\left(\mathrm{mmol} \mathrm{L}^{-1}\right)$ & $4.59 \pm 0.31$ & $4.45 \pm 0.30$ & $4.61 \pm 0.51$ & $3.81 \pm 0.16$ & $4.00 \pm 0.24$ & $3.70 \pm 0.08$ \\
\hline iCa $\left(\mathrm{mmol} \mathrm{L}^{-1}\right)$ & $1.53 \pm 0.05$ & $1.52 \pm 0.02$ & $1.48 \pm 0.06$ & $1.50 \pm 0.05$ & $1.50 \pm 0.02$ & $1.47 \pm 0.02$ \\
\hline Glu (mg dL $\left.{ }^{-1}\right)$ & $64.75 \pm 3.41$ & $69.13 \pm 4.32$ & $67.00 \pm 4.00$ & $100.33 \pm 5.43$ & $77.25 \pm 13.07$ & $84.50 \pm 13.20$ \\
\hline Het $(\% \mathbf{P C V})$ & $23.00 \pm 1.85$ & $22.88 \pm 1.34$ & $22.00 \pm 2.82$ & $19.67 \pm 2.07$ & $24.50 \pm 0.58$ & $20.75 \pm 3.30$ \\
\hline $\mathbf{H b}\left(\mathrm{g} \mathrm{dL}^{-1}\right)$ & $7.76 \pm 0.68$ & $7.16 \pm 0.46$ & $7.82 \pm 0.60$ & $6.65 \pm 0.67$ & $8.28 \pm 0.15$ & $7.05 \pm 1.12$ \\
\hline
\end{tabular}

\subsection{3. $\mathrm{P}_{3}$ period}

One day after ORP reached around $320-350 \mathrm{mV}$ at the $\mathrm{P}_{3}$ period, fish in RAS $\mathrm{O}_{3}$ began to show a appetite depression. Three days later, mortality occurred. During the $\mathrm{P}_{3}$ period, fish in RAS $\mathrm{O}_{3}$ exhibited a tired" behavior and responded slowly to stimulation. They could be divided into two populations: fish swimming in the upper water column and fish lying on the bottom of the tank. Once fish were lying down, they died 1-2 days later. Thus, only upper swimming ones were sampled for blood parameters including hematological parameters and plasma protein.

Compared with the RAS group, fish in the RAS $\mathrm{O}_{3} \mathrm{~S}$ group continued to show a higher $\mathrm{O}_{2}$, lower $\mathrm{CO}_{2}$, Hct and $\mathrm{Hb}$ concentrations and had a higher Glu concentration compared with fish of the same size in RAS (Table 7).

Two way ANOVA revealed that ORP significantly $(\mathrm{p}<0.05)$ impacted most hematological parameters, including $\mathrm{pH}, \mathrm{pCO}_{2}, \mathrm{pO}_{2}, \mathrm{HCO}_{3}, \mathrm{tCO}_{2}, \mathrm{sO}_{2}, \mathrm{~K}, \mathrm{Glu}$, Hct and $\mathrm{Hb}$ (Table 5). Fish size had significant effects $(\mathrm{p}<0.05)$ on $\mathrm{pCO}_{2}, \mathrm{Na}$, Glu and $\mathrm{Hb}$. Fish size and ORP interaction had significant effects $(\mathrm{p}<0.05)$ on $\mathrm{pH}, \mathrm{Glu}, \mathrm{Hb}$, etc. 
Table 7 : Hematological parameters of three size classes in RAS and RAS $\mathrm{O}_{3}$ during the $\mathrm{P}_{3}$ period

\begin{tabular}{|c|c|c|c|c|c|c|}
\hline \multirow[t]{2}{*}{ Parameters } & \multicolumn{2}{|c|}{$S 1$} & \multicolumn{2}{|c|}{$S 2$} & \multicolumn{2}{|c|}{$S 3$} \\
\hline & $R A S$ & $R A S O 3 S$ & $R A S$ & $R A S O 3 S$ & $R A S$ & RASO3S \\
\hline pH & $7.38 \pm 0.03$ & $7.38 \pm 0.02$ & $7.36 \pm 0.04$ & $7.47 \pm 0.03$ & $7.30 \pm 0.04$ & $7.41 \pm 0.05$ \\
\hline $\mathrm{pCO}_{2}\left(\mathrm{mmol} \mathrm{L}^{-1}\right)$ & $19.88 \pm 1.48$ & $18.40 \pm 0.42$ & $21.70 \pm 0.59$ & $15.40 \pm 1.19$ & $22.73 \pm 1.87$ & $15.55 \pm 1.49$ \\
\hline $\mathrm{pO}_{2}(\mathrm{mmHg})$ & $47.25 \pm 2.22$ & $53.00 \pm 6.78$ & $44.50 \pm 3.70$ & $53.50 \pm 4.20$ & $44.75 \pm 7.41$ & $69.75 \pm 11.79$ \\
\hline $\mathrm{HCO}_{3}\left(\mathrm{mmolL}^{-1}\right)$ & $11.93 \pm 1.14$ & $10.63 \pm 0.17$ & $11.95 \pm 0.73$ & $11.28 \pm 1.13$ & $11.28 \pm 1.61$ & $9.80 \pm 0.92$ \\
\hline $\mathrm{tCO}_{2}\left(\mathrm{mmol} \mathrm{L}^{-1}\right)$ & $12.50 \pm 1.00$ & $11.50 \pm 0.58$ & $13.00 \pm 0.82$ & $11.75 \pm 0.96$ & $12.00 \pm 1.83$ & $10.00 \pm 0.82$ \\
\hline $\mathrm{sO}_{2}(\%)$ & $84.25 \pm 2.63$ & $83.50 \pm 2.38$ & $75.25 \pm 3.50$ & $93.25 \pm 3.40$ & $78.75 \pm 3.74$ & $93.75 \pm 3.30$ \\
\hline $\mathrm{Na}\left(\mathrm{mmol} \mathrm{L} \mathbf{L}^{-1}\right)$ & $153.50 \pm 6.56$ & $158.75 \pm 1.50$ & $160.00 \pm 4.32$ & $161.00 \pm 0.82$ & $162.50 \pm 3.11$ & $161.25 \pm 3.95$ \\
\hline $\mathbf{K}\left(\mathbf{m m o l ~ L} \mathbf{L}^{-1}\right)$ & $4.20 \pm 0.83$ & $3.80 \pm 0.16$ & $4.13 \pm 0.35$ & $3.50 \pm 0.22$ & $4.23 \pm 0.31$ & $3.53 \pm 0.24$ \\
\hline iCa (mmol $\left.\mathrm{L}^{-1}\right)$ & $1.48 \pm 0.07$ & $1.42 \pm 0.09$ & $1.45 \pm 0.10$ & $1.52 \pm 0.03$ & $1.49 \pm 0.06$ & $1.49 \pm 0.06$ \\
\hline Glu (mg dL $\left.\mathbf{L}^{-1}\right)$ & $65.25 \pm 3.59$ & $76.50 \pm 3.32$ & $64.00 \pm 3.16$ & $123.00 \pm 17.90$ & $74.75 \pm 4.57$ & $90.75 \pm 15.72$ \\
\hline Het (\% PCV) & $23.50 \pm 1.91$ & $20.75 \pm 3.40$ & $23.25 \pm 2.63$ & $13.00 \pm 3.56$ & $24.00 \pm 2.83$ & $20.00 \pm 7.07$ \\
\hline$H b\left(g_{d L}{ }^{-1}\right)$ & $8.50 \pm 0.24$ & $7.40 \pm 0.22$ & $7.25 \pm 0.17$ & $4.75 \pm 1.15$ & $8.40 \pm 0.48$ & $7.28 \pm 1.54$ \\
\hline
\end{tabular}

\subsection{4. $\mathrm{P}_{4}$ period}

At the end of the $\mathrm{P}_{4}$ period, all groups in $\mathrm{RAS} \mathrm{O}_{3}$ had lower $\mathrm{PCO}_{2}, \mathrm{HCO}_{3}$, and $\mathrm{TCO}_{2}$ and higher $\mathrm{PO}_{2}$ and $\mathrm{SO}_{2}$ than the groups in RAS (Table 8). The increase in Glu concentration in $\mathrm{RAS}_{3}$ was higher with increasing fish size, and the level was higher in the $S_{2}$ and $S_{3}$ fish compared with the fish of the same size in RAS. Hb of $S_{2}$ and $S_{3}$ fish and $\mathrm{Hct}$ of $\mathrm{S}_{2}$ fish in RAS $\mathrm{O}_{3}$ were lower than those in RAS.

Two-way ANAOVA revealed that ORP had significant impacts on all hematological parameters except $\mathrm{Na}$ and iCa concentrations $(\mathrm{p}<0.05)$ (Table 5). Meanwhile fish size notably influenced $\mathrm{pO}_{2}, \mathrm{HCO}_{3}, \mathrm{tCO}_{2}, \mathrm{sO}_{2} \%, \mathrm{Na}, \mathrm{Glu}, \mathrm{Hb}$ and Hct. Fish size and ORP interactions significantly impacted $\mathrm{pH}, \mathrm{pCO}_{2}, \mathrm{pO}_{2}$, Glu, $\mathrm{Hct}$ and $\mathrm{Hb}$. 
Table 8: Hematological parameters of three size classes in RAS and RAS $\mathrm{O}_{3}$ during the $\mathrm{P}_{4}$ period

\begin{tabular}{|c|c|c|c|c|c|c|}
\hline \multirow[t]{2}{*}{ Parameters } & \multicolumn{2}{|l|}{$S 1$} & \multicolumn{2}{|l|}{$S 2$} & \multicolumn{2}{|l|}{$S 3$} \\
\hline & $\boldsymbol{R A S}$ & $R A S \quad O 3$ & $\boldsymbol{R A S}$ & $\mathrm{RASO3}$ & $\boldsymbol{R A S}$ & $\mathrm{RASO3}$ \\
\hline pH & $7.39 \pm 0.06$ & $7.38 \pm 0.03$ & $7.45 \pm 0.03$ & $7.41 \pm 0.03$ & $7.34 \pm 0.01$ & $7.46 \pm 0.05$ \\
\hline $\mathrm{pCO}_{2}\left(\mathrm{mmol} \mathrm{L}^{-1)}\right.$ & $25.78 \pm 2.06$ & $16.25 \pm 1.67$ & $25.95 \pm 0.65$ & $16.05 \pm 0.47$ & $27.38 \pm 0.39$ & $12.38 \pm 0.42$ \\
\hline $\mathrm{pO}_{2}(\mathrm{mmHg})$ & $45.00 \pm 6.27$ & $61.25 \pm 8.00$ & $54.00 \pm 2.45$ & $88.75 \pm 2.99$ & $47.25 \pm 3.59$ & $94.00 \pm 1.63$ \\
\hline $\mathrm{HCO}_{3}\left(\mathrm{mmolL}^{-1}\right)$ & $16.78 \pm 0.43$ & $10.08 \pm 0.51$ & $15.03 \pm 1.31$ & $9.60 \pm 0.80$ & $14.18 \pm 0.24$ & $8.88 \pm 1.11$ \\
\hline $\mathrm{tCO}_{2}\left(\mathrm{mmol} \mathrm{L}^{-1}\right)$ & $17.50 \pm 1.73$ & $10.50 \pm 0.58$ & $15.75 \pm 1.70$ & $10.50 \pm 1.00$ & $15.50 \pm 0.58$ & $8.75 \pm 0.96$ \\
\hline $\mathrm{sO}_{2}(\%)$ & $77.25 \pm 9.43$ & $87.50 \pm 1.00$ & $86.75 \pm 1.70$ & $94.00 \pm 4.24$ & $81.00 \pm 3.16$ & $94.25 \pm 3.20$ \\
\hline $\mathrm{Na}\left(\mathrm{mmol} \mathrm{L}^{-1}\right)$ & $158.75 \pm 4.35$ & $160.75 \pm 1.70$ & $162.25 \pm 0.96$ & $160.25 \pm 4.1$ & $164.50 \pm 2.38$ & $163.00 \pm 1.83$ \\
\hline $\mathrm{K}\left(\mathrm{mmol} \mathrm{L} \mathbf{L}^{-1}\right)$ & $3.88 \pm 0.26$ & $3.48 \pm 0.19$ & $3.95 \pm 0.19$ & $3.68 \pm 0.22$ & $4.08 \pm 0.26$ & $3.28 \pm 0.24$ \\
\hline iCa $\left(\mathrm{mmol} \mathrm{L}^{-1}\right)$ & $1.50 \pm 0.03$ & $1.49 \pm 0.05$ & $1.46 \pm 0.02$ & $1.49 \pm 0.03$ & $1.50 \pm 0.03$ & $1.50 \pm 0.03$ \\
\hline Glu $\left(\mathrm{mg} \mathrm{dL}^{-1}\right)$ & $76.25 \pm 8.88$ & $79.75 \pm 4.11$ & $70.5 \pm 2.38$ & $88.75 \pm 2.99$ & $70.25 \pm 1.50$ & $105.75 \pm 13.33$ \\
\hline Het (\% PCV) & $23.75 \pm 2.75$ & $20.50 \pm 2.52$ & $23.75 \pm 2.87$ & $14.00 \pm 2.45$ & $23.50 \pm 2.38$ & $21.00 \pm 2.16$ \\
\hline $\mathrm{Hb}\left(\mathrm{g} \mathrm{dL}^{-1}\right)$ & $7.93 \pm 0.51$ & $7.30 \pm 0.33$ & $8.30 \pm 0.58$ & $4.78 \pm 0.83$ & $8.78 \pm 0.19$ & $7.05 \pm 0.56$ \\
\hline
\end{tabular}

\subsection{Protein concentration in plasma}

At the end of $\mathrm{P}_{3}$ period, RAS $\mathrm{O}_{3}$ had a lower plasma protein concentration compared with RAS(Fig. 3).

The two-way ANOVA analysis showed that ORP significantly impacted $(p<0.05)$ plasma protein concentrations of fish in RAS $\mathrm{O}_{3}$ since the $\mathrm{P}_{2}$ period. At the end of $\mathrm{P}_{3}$ period, the significant influence of ORP $(p<0.05)$ on the protein concentration continued. At the same time, fish size also had a significant influence $(p<0.05)$ on the plasma protein concentration. While at the $\mathrm{P}_{4}$ period, no significant impact $(p>0.05)$ of fish size and ORP on the plasma protein was found. 

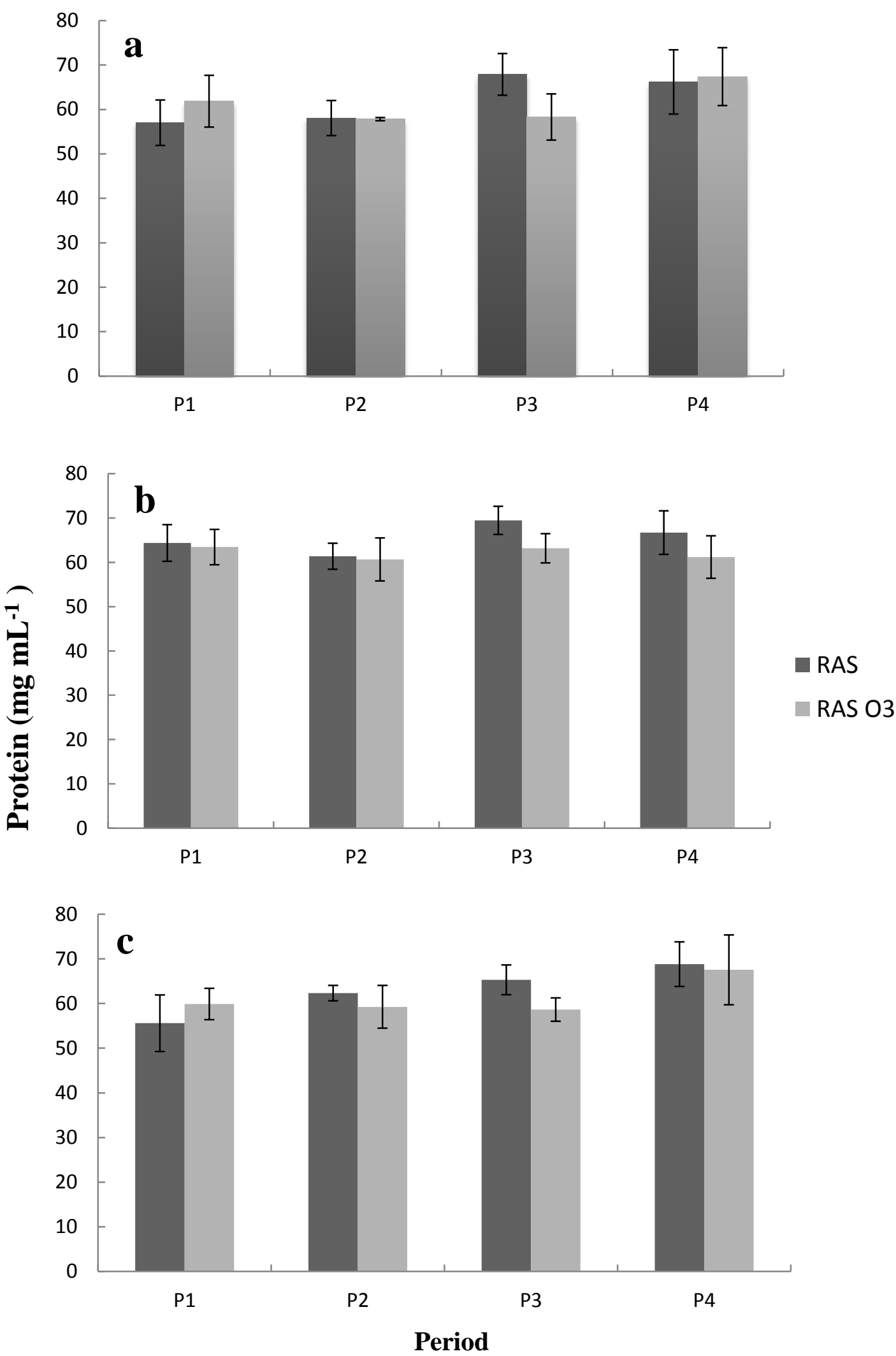

Fig. 3a-c : Plasma protein concentration ( $\mathrm{mg} \mathrm{ml-1}$ ) of $\mathrm{S}_{1}$ : small size (a) and $\mathrm{S}_{2}$ : medium size (b) and $\mathrm{S}_{3}$ : large size (c) in RAS and RAS $\mathrm{O}_{3}$ during four periods 
Table 9: Two-way ANOVA analysis of ORP, fish size and ORP* fish size on plasma protein

\begin{tabular}{|c|c|c|c|c|}
\hline Indices & P1 & P2 & P3 & P4 \\
\hline ORP & + & ++ & +++ & + \\
\hline Fish Size & + & + & ++ & + \\
\hline ORP*Fish Size & + & + & + & + \\
\hline
\end{tabular}

+ : difference is not significant $(\mathrm{p}>0.05)$; ++ : difference is significant $(0.01 \leq \mathrm{p}<0.05) ;+++$ : difference is extremely $(\mathrm{p}<0.01)$

\section{Discussion}

\subsection{Water parameters}

It is noticeable that ORP levels and TRO concentrations measured in this study were not consistent with those reported from previous studies, likely due to the use of different ORP probes and their locations.

In a freshwater rainbow trout RAS, the ORP measured in the fish tank drain using a platinum electrode was 155-200 mV, without ozonation (Davidson et al. 2011). It was $220 \mathrm{mV}$ in a marine Atlantic halibut RAS without ozone (Tango and Gagnon 2003). In a marine sea bream RAS, ORP was $\sim 240 \mathrm{mV}$ without ozone in the foam fraction (Park et al. 2011). In a lobster hatchery without ozone, ORP was $350 \mathrm{mV}$ (Jensen et al. 2011). In the current study, the ORP level measured in fish tanks of the RAS, using ODEON probes calibrated once a week on average was $250-270 \mathrm{mV}$. Thus, it is not easy to compare the ORP level among different RAS because of differences in seawater parameters, aquaculture operations, and probes (Liu et al. 2009). Moreover, it would take $2 \mathrm{~h}$ to obtain the ORP equilibrium value when salinity is $30 \mathrm{ppt}$ (Wang et al. 2011). Overall, ORP measurement remains a challenging issue.

Ozone effectively oxidizes ammonia, and nitrite degrades yellow substances and modifies the biochemical oxygen demand and organic carbon concentration in freshwater or marine RAS (Davidson et al. 2011; Schroeder et al. 2011; Summerfelt et al. 1997; Summerfelt et al. 2009). In an ozonated marine Atlantic halibut RAS, when ORP reached $320-340 \mathrm{mV}$ in the biofilters, it resulted in a $15 \%$ reduction of total organic carbon and a reduction in nitrite, color, and suspended solids compared to the control RAS with an ORP of $\sim 220 \mathrm{mV}$ (Tango and Gagnon 2003).

No significant benefit of ozonation on ammonia or nitrite reduction was detected in the current study. This was likely because the fish biomass was far from the carrying capacity of the biofilters. Water in both systems showed a suitable quality for sea bass. However, there was an increase in TSS in RAS $\mathrm{O}_{3}$ during the $\mathrm{P}_{3}$ period, and this was attributed to accumulation of uneaten feed in the system. TSS in RAS $\mathrm{O}_{3}$ during the $\mathrm{P}_{4}$ 
period decreased, likely because the fish exhibited decreased appetite. Parallel experiments, carried out on the microbial flora of both the rearing water and the biofilter media, revealed a moderate impact of ORP on bacterial abundance, activity and diversity (data not shown).

\subsection{Mortality}

Once ORP exceeded $320 \mathrm{mV}$ during the $\mathrm{P}_{3}$ period, fish appetite decreased. Three days later, first mortality occurred. After the ORP was decreased to 300-320 mV during the $\mathrm{P}_{4}$ period, mortality continued. Most previous studies related to ozonation toxicity were carried out on fresh water species or focused on the acute response (Bullock et al. 1997; Fukunaga et al. 1992; Good et al. 2011). Rarely data are available for European sea bass. Reiser et al (2010) suggested concentrations of $\leq 0.06 \mathrm{mg} \mathrm{L}-1$ as acceptable OPO levels for turbot juveniles. For white perch, gill cell damage was observed at an OPO concentration of $0.05 \mathrm{mg} \mathrm{L}^{-1}$ for $96 \mathrm{~h}$, and LC-50 (lethal concentration leading to $50 \%$ death) for adult white perch was around $0.2 \mathrm{mg} \mathrm{L}^{-1}$ (Richardson et al. 1983). For Paralichthys olivaceus, 48 h LC-50 was around $0.13 \mathrm{mg}$ $\mathrm{L}^{-1}$ (Jiang et al. 2001). Therefore, the mortality, which occurred two days after ORP reached $320-350 \mathrm{mV}$ in the RAS $\mathrm{O}_{3}$ fish tanks at the $\mathrm{P}_{3}$ period is to be attributed to the ORP and not to TRO concentration.

In addition, the mortality continued at the $\mathrm{P}_{4}$ period, even when the ORP level fell to the same level as it was at the $P_{2}$ period. It seems that the damage to fish health caused by too high ORP levels is not easy to be recovered.

In conclusion, even a short-term too high ORP in fish tanks should be avoided in aquaculture systems.

\subsection{Hematological parameters}

In this study, the hematological parameters was accordance with data reported from Sammouth et al. 2009. Hematological parameters are widely used to evaluate toxic and chemical stress and to determine the health status of fish (Saravanan et al. 2011). The alterations of iCa and $\mathrm{K}$ that were induced by ORP indicated an adjustment of metabolism in blood cells.

$\mathrm{Hb}$ is the protein in red blood cells that delivers oxygen to the organs, and Hct describes the percent of red blood cells. $\mathrm{Hb}$ and $\mathrm{Hct}$ were significantly affected by ORP levels over the $P_{1}, P_{2}, P_{3}$ and $P_{4}$ period. $A$ decreased $\mathrm{Hb}$ and $\mathrm{Hct}$ and a blood gas balance modification (higher blood $\mathrm{O}_{2}$ and lower $\mathrm{CO}_{2}$ ) was measured in the RAS $\mathrm{O}_{3}$ seabass compared with same size fish in the RAS. A two-way ANOVA analysis revealed that the ORP level significantly affected the gas balance in the blood. At the end of the $\mathrm{P} 1$ period, $\mathrm{sO}_{2} \%$ was significantly influenced by ORP. During $\mathrm{P} 2$ period it affected significantly most blood gas parameters including $\mathrm{pCO}_{2}, \mathrm{pO}_{2}, \mathrm{HCO}_{3}, \mathrm{tCO}_{2}$, 
and $\mathrm{sO}_{2} \%$. It seems that ORP above $300-320 \mathrm{mV}$ would negatively influence the seabass hematological parameters. There is available information on the relationship between ORP levels and fish physiology. It seems that fish species react differently to ozonation. Silva et al (2011) reported the increased red blood cell and Hct concentrations in turbot juveniles exposed to ozonated seawater, should be regarded as adaptation to TRO rather than to toxicity. For rainbow trout in an ozonated $\mathrm{RAS}, \mathrm{Hb}$ and Hct were slightly lower than in fish in an RAS without ozone, but the difference was not significant (Good et al. 2011). Fukunaga et al $(1992,1999)$ suggested that, the decrease of $\mathrm{Hct}$ and $\mathrm{Hb}$ in the red blood cells of fish exposed to ozone, corresponded to liquid peroxidation and hemolysis of the cell membrane. In this study, TRO remained lower than $0.02 \mathrm{mg} \mathrm{L}-1$ at the $\mathrm{P}_{1}, \mathrm{P}_{2}$ and $\mathrm{P}_{4}$ period, and the concentration of 0.03-0.05 measured at the $\mathrm{P}_{3}$ period was below the toxic dose. Therefore the decreased $\mathrm{Hb}$ and Hct observed in our experiment corresponded likely to the long-term adaption of the fish to the modified ORP and not to the toxicity of TRO on red blood cell. Since the oxygen concentration of fish tanks in RAS and RAS $\mathrm{O}_{3}$ were similar, the influence of ORP on the gas balance and the function of related blood cells in the fish need to be investigated further.

Acid-base regulation in fish is complex and involves a series of reactions for regulating $\mathrm{HCO}_{3}{ }^{-}$and $\mathrm{H}^{+}$concentrations in the blood. In this study, blood $\mathrm{pH}$ was also significantly affected by the ORP levels at the end of $\mathrm{P}_{2}, \mathrm{P}_{3}$ and $\mathrm{P}_{4}$ periods, and corresponded to a gas balance modification in the blood.

Most of living organism's energy comes from carbohydrate transformation, which increases to meet energy demand during stress situations. It is well acknowledged that the blood glucose levels are closely correlated to the level of stress of fish (Endo et al. 2006). In this study, the glucose concentration was significantly influenced by the ORP levels since the $\mathrm{P}_{2}$ period (ORP between $300-320 \mathrm{mV}$ ). Glu was higher in $\mathrm{RAS}_{3}$ than in the same size fish in RAS. Same findings were reported by Maricchiolo et al (2011) when seabass were stressed. As 300-320 mV ORP started to stress seabass, it seems that the blood glucose could be a good indicator for ORP stress.

\subsection{Protein concentration}

During the entire experimental period, the plasma protein ranged between 55 and 70 $\mathrm{mg} \mathrm{ml}^{-1}$, which is accordance with data reported from a previous study (Sammouth et al. 2009). At the end of $P_{2}$ and $P_{3}$ periods, protein concentration was significantly influenced by ORP, indicating that ORP around 300-320 mV started to stress seabass. During $\mathrm{P}_{3}$ period when ORP rose to $320-350 \mathrm{mV}$ in the fish tanks, fish appetite was depressed and mortality occurred. As a consequence, protein in plasma decreased due to the lack of nutrients and impaired health. The increase of plasma protein measured when seabass recovered from a hydrogen peroxide exposure (Roque et al. 2010) was not observed in our experiment. 


\section{Conclusion}

The results of the present study showed that several hematological parameters were altered by increased ORP levels. Blood glucose and plasma protein concentration alterations indicated that ORP above 300-320 started to stress seabass. Short term exposure to ORP levels around 320-350 induced mortality. Therefore it is strongly recommended that that the ORP level, which is increased by the use of strong oxidants as ozone, should be maintained below $320 \mathrm{mV}$ for European sea bass rearing in RAS.

Further studies are necessary to identify the best ORP level for European sea bass grow out, and to investigate how ORP alters the physiological fish parameters.

\section{Acknowledgements}

The authors would thank all the participants from the Ifremer Palavas station: Cyrille Przybyla, Myriam Callier and Thibault Geoffroy for their contribution to the experiment and analyses. This work was supported by the National Natural Science Foundation of China (grant no.41306152) and National Science and Technology Support Program(grant no.2011BAD13B04).

This article is also a part of cooperation between China (IOCAS), France (Ifremer) and Italy (University of Messina).

\section{References}

Banhidi M (1995) pH and ORP. Met Finish 93: 544-550

Blancheton JP (2000) Developments in recirculation systems for Mediterranean fish species. Aquacult Eng 22: 17-31

Buchan KAH, Martin-Robichaud DJ, Benfey TJ (2005) Measurement of dissolved ozone in sea water: A comparison of methods. Aquacult Eng 33: 225-231

Buchan KAH, Martin-Robichaud DJ, Benfey TJ, MacKinnon AM, Boston L (2006) The efficacy of ozonated seawater for surface disinfection of haddock (Melanogrammus aeglefinus) eggs against piscine nodavirus. Aquacult Eng 35: 102-107

Bullock GL, Summerfelt ST, Noble AC, Weber AL, Durant MD, Hankins JA (1997) Ozonation of a recirculating rainbow trout culture system I. Effects on bacterial gill disease and heterotrophic bacteria. Aquaculture 158: 43-55

Caldwell CA, Hinshaw J (1994) Physiological and haematological responses in rainbow trout subjected to supplemental dissolved oxygen in fish culture. Aquaculture 126: 183-193 
Davidson J, Good C, Welsh C, Summerfelt S (2011) The effects of ozone and water exchange rates on water quality and rainbow trout Oncorhynchus mykiss performance in replicated water recirculating systems. Aquacult Eng 44: 80-96

Endo H, Yonemori Y, Musiya K, Maita M, Shibuya T, Ren H, Hayashi T, Mitsubayashi $\mathrm{K}$ (2006) A needle-type optical enzyme sensor system for determining glucose levels in fish blood. Analytica Chimica Acta 22: 573-574

Forneris G, Bellardi S, Palmegiano GB, Saroglia M, Sicuro B, Gasco L, Zoccarato I (2003) The use of ozone in trout hatchery to reduce saprolegniasis incidence. Aquaculture 221: 157-166

Fukunaga K, Nakazono N, Suzuki T, Takama K (1999) Mechanism of oxidative damage to fish red blood cells by ozone. lubmb Life 48: 631-634

Fukunaga K, Suzuki T, Arita M, Suzuki S, Hara A, Yamauchi K, Shinriki N, Ishizaki K, Takama K (1992) Acute Toxicity of Ozone against Morphology of Gill and Erythrocytes of Japanese Charr (Salvelinus-Leucomaenis). Comp Biochem Phys C 101: 331-336

Good C, Davidson J, Welsh C, Snekvik K, Summerfelt S (2011) The effects of ozonation on performance, health and welfare of rainbow trout Oncorhynchus mykiss in low-exchange water recirculation aquaculture systems. Aquacult Eng 44: 97-102

Jensen MA, Ritar AJ, Burke C, Ward LR (2011) Seawater ozonation and formalin disinfection for the larval culture of eastern rock lobster, Jasus (Sagmariasus) verreauxi, phyllosoma. Aquaculture 318: 213-222.

Jiang G, Liu Y, Yang D, Lue $Y$ (2001) The toxicity of ozonated seawater to the Penaeus chinensis and Paralichthys olivaceus. Marine Sciences 25 (3): 11-13.

Kristensen T, Åtland Å, Rosten T, Urke HA, Rosseland BO (2009) Important influent-water quality parameters at freshwater production sites in two salmon producing countries. Aquacult Eng 41: 53-59

Liu XQ, Wang J, Zhang D, Li YT (2009) Grey relational analysis on the relation between marine environmental factors and oxidation-reduction potential. Chin $\mathrm{J}$ Oceanol Limnol 27: 583-586

Maricchiolo G, Mirto S, Caruso G, Caruso T, Bonaventura R, Celi M, Matranga V, Genovese L, 2011. Welfare status of cage farmed European sea bass (Dicentrarchus labrax): A comparison between submerged and surface cages. Aquaculture 314: 173-181

Park J, Kim Y, Kim PK, Daniels HV (2011) Effects of two different ozone doses on seawater recirculating systems for black sea bream Acanthopagrus schlegeli (Bleeker): Removal of solids and bacteria by foam fractionation. Aquacult Eng 44: 19-24

Reiser S, Schroeder JP, Wuertz S, Kloas W, Hanel R (2010) Histological and physiological alterations in juvenile turbot (Psetta maxima, L.) exposed to sublethal concentrations of ozone-produced oxidants in ozonated seawater. Aquaculture 307: 157-164 
Richardson LB, Burton DT, Block RM, Stavola AM (1983) Lethal and sublethal exposure and recovery effects of ozone-produced oxidants on adult white perch (Morone americana Gmelin). Water Res 17: 205-213

Ritar AJ, Smith GG, Thomas CW (2006) Ozonation of seawater improves the survival of larval southern rock lobster, Jasus edwardsii, in culture from egg to juvenile. Aquaculture 261: 1014-1025

Roque A, Yildiz HY, Carazo I, Duncan N (2010) Physiological stress responses of sea bass (Dicentrarchus labrax) to hydrogen peroxide $(\mathrm{H} 2 \mathrm{O} 2)$ exposure. Aquaculture 304: 104-107

Sammouth S, d'Orbcastel ER, Gasset E, Lemarié G, Breuil G, Marino G, Coeurdacier JL, Fivelstad S, Blancheton JP (2009) The effect of density on sea bass (Dicentrarchus labrax) performance in a tank-based recirculating system. Aquacult Eng 40: 72-78

Sanni S, Forsberg Ol (1996) Modelling $\mathrm{pH}$ and carbon dioxide in single-pass sea-water aquaculture systems. Aquacult Eng 15: 91-110

Saravanan M, Prabhu Kumar K, Ramesh M (2011) Haematological and biochemical responses of freshwater teleost fish Cyprinus carpio (Actinopterygii: Cypriniformes) during acute and chronic sublethal exposure to lindane. Pestic Biochem Phys 100: 206-211

Schroeder JP, Croot PL, Von Dewitz B, Waller U, Hanel R (2011) Potential and limitations of ozone for the removal of ammonia, nitrite, and yellow substances in marine recirculating aquaculture systems. Aquacult Eng 45: 35-41

Silva J, Laranjeira A, Serradeiro R, Santos MA, Pacheco M (2011) Ozonated seawater induces genotoxicity and hematological alterations in turbot (Scophthalmus maximus) - Implications for management of recirculation aquaculture systems. Aquaculture 318: 180-184

Smith PK, Krohn RI, Hermanson GT, Mallia AK, Gartner FH, Provenzano MD, Fujimoto EK, Goeke NM, Olson BJ, Klenk DC (1985) Measurement of protein using bicinchoninic acid. Anal Biochem 150: 76-85

Soria G, Merino G, von Brand E (2007) Effect of increasing salinity on physiological response in juvenile scallops Argopecten purpuratus at two rearing temperatures. Aquaculture 270: 451-463

Summerfelt ST, Hankins JA, Weber AL, Durant MD (1997) Ozonation of a recirculating rainbow trout culture system II. Effects on microscreen filtration and water quality. Aquaculture 158: 57-67

Summerfelt ST, Sharrer MJ, Tsukuda SM, Gearheart M (2009) Process requirements for achieving full-flow disinfection of recirculating water using ozonation and UV irradiation. Aquacult Eng 40: 17-27

Tal Y, Schreier HJ, Sowers KR, Stubblefield JD, Place AR, Zohar Y (2009) Environmentally sustainable land-based marine aquaculture. Aquaculture 286:28-35

Tango MS, Gagnon GA (2003) Impact of ozonation on water quality in marine recirculation systems. Aquacult Eng 29: 125-137 
Thorarensen H, Gústavsson Aó, Mallya Y, Gunnarsson S, Árnason J, Arnarson I, Jónsson AF, Smáradóttir H, Zoega GT, Imsland AK (2010) The effect of oxygen saturation on the growth and feed conversion of Atlantic halibut (Hippoglossus hippoglossus L.). Aquaculture 309: 96-102

Wang Y, Liu DX, Ju MT, Jin ZH, Li TL (2011) The effect of seawater salinity on the equilibrium time of oxidation reduction potential. Adv Mater Res 301: 1648-1651

Whitfield M (1974) The hydrolysis of ammonium ions in sea water-a theoretical study. J Mar Biol Assoc UK 54: 565-580

Zhang SY, Li G, Wu HB, Liu XG, Yao YH, Tao L, Liu H (2011) An integrated recirculating aquaculture system (RAS) for land-based fish farming: The effects on water quality and fish production. Aquacult Eng 45: 93-102 